\title{
Local management after neoadjuvant treatment for breast cancer
}

\author{
Takehiko Sakai, Takayuki Ueno \\ Department of Surgical Oncology, Breast Oncology Center, Cancer Institute Hospital of JFCR, Tokyo, Japan \\ Contributions: (I) Conception and design: All authors; (II) Administrative support: T Ueno; (III) Provision of study materials or patients: T Sakai; \\ (IV) Collection and assembly of data: All authors; (V) Data analysis and interpretation: All authors; (VI) Manuscript writing: All authors; (VII) Final \\ approval of manuscript: All authors. \\ Correspondence to: Takehiko Sakai. 3-8-31 Ariake, Koto-ku, Tokyo 135-8550, Japan. Email: takehiko.sakai@jfcr.or.jp.
}

\begin{abstract}
Neoadjuvant chemotherapy (NAC) was originally used in patients with locally advanced breast cancer. Then, it is used in operable breast cancer to downstage the primary breast cancer and axillary lymph nodes metastasis, result in improving the cosmetic outcome and decreasing surgical morbidity. However, it is sometimes difficult to assess the extent of residual disease after NAC, as the NAC reduces the lesion and obscure the original images both breast and axilla. Thus, detailed assessment of primary breast cancer and axillary lymph nodes metastasis are required from the time of before NAC until the time of surgery. These assessments include the accurate location, the extent of intraductal component around primary tumor and the axillary nodal status. Multimodality imaging with intervention for cytopathology can help to delineate the size and location of breast cancer and lymph node metastasis and predict the residual tumor burden in primary breast cancer and involved axillary nodes. In the future, with development of new targeted therapy, technologies in medical imaging diagnosis and ongoing trial data will provide further individualized treatment option for patients with breast cancer. This article reviews the current evidence and management recommendations for optimal surgical treatment in this setting.
\end{abstract}

Keywords: Neoadjuvant chemotherapy (NAC); local treatment; breast cancer; assessment of breast; assessment of axillary nodal status

Submitted Jun 02, 2020. Accepted for publication Jun 19, 2020.

doi: $10.21037 / \mathrm{cco}-20-164$

View this article at: http://dx.doi.org/10.21037/cco-20-164

\section{Introduction}

Traditionally, neoadjuvant treatment, which most often applied as neoadjuvant chemotherapy (NAC) before surgery, was used in patients with locally advanced breast cancer to convert them to operable disease. But NAC for early breast cancer patients is now recognized as a useful clinical tool for some reasons, the potential for converting patients requiring mastectomy to candidate for breastconserving surgery (BCS), the potential for converting patients requiring axillary dissection to candidates for sentinel lymph node biopsy alone. Furthermore, tumor response to neoadjuvant treatment is an important prognostic factor and surrogate marker for overall survival and useful for response-guided treatment strategy at least for some subtypes of breast cancer (1). According to two prospective trials, von Minckwitz et al. (2) and Masuda et al. (3) reported that the additional treatment can improve long-term outcomes for patients who have not achieved best response with NAC and targeted therapy, who are at high risk for recurrence. The information of tumor response will guide postoperative systemic therapy.

There has been great improvement in pathologic complete response (pCR) rates in the past decade, especially in human epidermal growth factor receptor 2 (HER2)positive and triple-negative breast cancer. In patients with hormone receptor-negative and HER2-positive, pCR were noted in $63 \%$ women who received both anti-HER2 therapy and chemotherapy (4). With improved mortality of breast cancer, the quality of life of breast cancer survivor has become more important and attention has been focused on less-invasive, patient-friendly local treatment. In the 
future, novel targeted therapy and technology will lead to further individualization of local treatment for breast cancer, including the possibility of no surgery in cases with achieving a good response. This article reviews the current topics surrounding surgery of the breast and axilla in patients with breast cancer receiving neoadjuvant treatment.

\section{Breast surgery after NAC}

One of the purposes of NAC from a surgical viewpoint is improving the cosmetic quality of life following BCS by reducing the tumor size even if the patient is a candidate for conserving surgery at diagnosis. A meta-analysis of 10 randomized controlled trials of neoadjuvant versus adjuvant chemotherapy demonstrated that patients allocated NAC were more likely to have BCS (65\% vs. 49\%) (5). Although there is not significant difference in distant recurrence between NAC and adjuvant chemotherapy (15 year risk $38 \%$ for NAC vs. $38 \%$ for adjuvant chemotherapy; $\mathrm{P}=0.66$ ), $\mathrm{NAC}$ was associated with more frequent local recurrence than was adjuvant chemotherapy (15 year risk $21 \%$ for NAC vs. $16 \%$ for adjuvant chemotherapy; $\mathrm{P}=0.0001$ ) (5). When considering the cause of this local failure, it is probably due to the difficulty in assessing the extent of residual disease after NAC.

\section{Assessment of breast cancer during NAC}

It is crucial for appropriate BCS to assess accurately the location and extent of the primary breast cancer with or without NAC. It could be often difficult to accurately assess the extent of the lesion, as the NAC reduces the lesion and obscure the original images. Thus, assessment of primary breast cancer from the time of diagnosis until the time of surgery is most important for controlling local managements. Physical examination, mammography, ultrasonography, and magnetic resonance imaging (MRI) can help to delineate the size and configuration of the tumor. Mammography can delineate the extent of malignant calcifications indicating an extent of intraductal component around primary tumor (6). MRI can also help to assess the extent of breast cancer and their response to NAC. Several studies have demonstrated the accuracy of MRI compared with physical examination, mammography, and ultrasonography in determining the presence and extent of viable cancer within the breast after NAC $(7,8)$. Nakamura classified the patterns of breast tumor growth and shrinkage into concentric or dendritic pattern and concentric pattern was good candidates for BCS after NAC (9).
Ultrasonography is useful for drawing a map of the excision area on the body surface because the patient can be examined in the same supine position as surgery. It is paramount to perform these multimodality imaging before, during and after NAC when aiming for BCS. Although these imaging provides further information about the breast cancer, there are various differences in the imaging equipment, interpretation and surgical method, handling of BCS specimens and pathological diagnosis $(10,11)$. Well-coordinated multidisciplinary oncology team is necessary to discuss and improve the correctness of their management based on the pathological result, short- and long-term outcomes.

\section{No surgery (active surveillance) after NAC}

With improvements in new targeted therapy and chemotherapy regimens, pCR rate after NAC has increased over the last decade, specifically in triple-negative and HER2 positive breast cancer. Up to $40 \%$ of patients with HER2 positive who had given dual anti-HER2 therapy (trastuzumab plus pertuzuma) with docetaxel achieved pCR (4). If pCR has achieved to a patient, theoretically she may not need surgery at all. According to Özkurt et al. (12), one of the largest retrospective cohort study in the U.S., demonstrated that achieving a complete response after NAC may have a favorable outcome on patients OS, even in patients not undergoing local therapy to the breast. Although in a meta-analysis in a previous era by Mauri et al. (13) demonstrated the patients who adopted radiotherapy without surgery had a significantly increased risk of loco-regional recurrences, high pCR rates in the modern era and advanced pCR prediction methods using breast imaging with biopsy might enable the strategy of "No surgery" after NAC. Inserting a radiopaque marker under mammography or ultrasonography is recommended for some patients who have a potential to achieve pCR (14). Several groups have begun accrual on the trials to investigate the possibility of no surgery after NAC (15-17). The study ongoing in MD Anderson Cancer Center is designed to evaluate whether patients with residual carcinoma can be accurately identified using image-guided intervention (17). Safe omission of surgery in patients after NAC is depend on the accurate assessment of breast and axillary tumor burden.

\section{Axillary management after NAC}

The criteria for clinically node-negative disease is defined as no regional node metastases (by imaging or clinical 
examination) in AJCC Cancer Staging manual, eighth edition. In the setting of NAC, imaging diagnosis is recommended for evaluation of axillary lymph nodes before NAC as baseline (18). Several reports have demonstrated the high diagnostic sensitivity of axillary lymph node evaluation by means of ultrasonography (19), CT $(20,21)$, MRI (22) or EDG-PET. Although the diagnostic sensitivity with these modalities is higher than palpation, axillary ultrasonography and ultrasonography guided FNA or CNB of abnormal lymph nodes is minimally invasive intervention that can assess axillary lymph node repeatedly and provide direct information of the chemosensitivity of axillary metastases to NAC $(19,23)$.

In $\mathrm{cN} 0$ patients at baseline, SNB might be performed after NAC considering its accuracy with a low false negative rate (FNR) (24) and recommendations of updated guidelines by the National comprehensive cancer Network (NCCN) and Japanese Breast Cancer Society (25).

For patients with 1 or more cytology/histologyproven positive nodes at baseline, axillary dissection has been the standard treatment in these patients according to initial studies that yielded an FNR $>10 \%(26,27)$. Three prospective trials (SN FNAC, Z1071, SENTINA) addressing the feasibility of SLN after NAC in patients with clinically node-positive disease have demonstrated the lower FNR $<10 \%$ in selected patients $(23,28,29)$ when more than 2 lymph nodes were removed, dual agent were used for SLN mapping and positive lymph nodes were marked with a US-visible marker before starting NAC. These results suggest SNB is accurate if three or more sentinel lymph nodes are evaluated, but long-term outcome date from patients treated with SNB alone in this setting is lacking. Patients in this setting should be discussed at a multidisciplinary meeting to evaluate if axillary dissection can be omitted or should be recommended.

For patients with a large axillary lymph node involvement before NAC or for those who have residual disease in the axilla after NAC, standard therapy of the axilla is axillary dissection. However, detailed assessment of axillary lymph nodes by imaging and cyto-pathological intervention will probably provide more tailored management of axilla. Some prospective trials opened to investigate the role of SLN and regional lymph nodes radiation in patients with $\mathrm{cN} 1$ after NAC (30).

\section{Conclusions}

The local management of early breast cancer patients after NAC will probably be required more individualized approach based on the preoperative treatment response. Multimodality imaging with intervention for cytopathology can help to delineate the size and location of breast cancer and lymph node metastasis and predict the residual tumor burden in primary breast cancer and involved axillary nodes. In the future, with development of new targeted therapy, technologies in medical imaging diagnosis and ongoing trial data will provide further individualized treatment option for patients with breast cancer.

\section{Acknowledgments}

Funding: None.

\section{Footnote}

Provenance and Peer Review: This article was commissioned by the Guest Editors (Yutaka Yamamoto and Takayuki Ueno) for the series "Neoadjuvant/Adjuvant Treatment for Early Breast Cancer" published in Chinese Clinical Oncology. The article was sent for external peer review organized by the Guest Editors and the editorial office.

Conflicts of Interest: Both authors have completed the ICMJE uniform disclosure form (available at http://dx.doi. org/10.21037/cco-20-164). The series "Neoadjuvant/ Adjuvant Treatment for Early Breast Cancer" was commissioned by the editorial office without any funding or sponsorship. TU served as the unpaid Guest Editor of the series. TU reports personal fees from Chugai Pharmaceutical Co., Ltd., grants and personal fees from Eisai Co., Ltd., personal fees from AstraZeneca, personal fees from Taiho, personal fees from Novartis Pharma K.K., during the conduct of the study. The other author has no other conflicts of interest to declare.

Ethical Statement: The authors are accountable for all aspects of the work in ensuring that questions related to the accuracy or integrity of any part of the work are appropriately investigated and resolved.

Open Access Statement: This is an Open Access article distributed in accordance with the Creative Commons Attribution-NonCommercial-NoDerivs 4.0 International License (CC BY-NC-ND 4.0), which permits the noncommercial replication and distribution of the article with the strict proviso that no changes or edits are made and the 
original work is properly cited (including links to both the formal publication through the relevant DOI and the license). See: https://creativecommons.org/licenses/by-nc-nd/4.0/.

\section{References}

1. Cortazar P, Zhang L, Untch M, et al. Pathological complete response and long-term clinical benefit in breast cancer: the CTNeoBC pooled analysis. Lancet 2014;384:164-72.

2. von Minckwitz G, Huang CS, Mano MS, et al. Trastuzumab Emtansine for Residual Invasive HER2Positive Breast Cancer. N Engl J Med 2019;380:617-28.

3. Masuda N, Lee SJ, Ohtani S, et al. Adjuvant Capecitabine for Breast Cancer after Preoperative Chemotherapy. N Engl J Med 2017;376:2147-59.

4. Gianni L, Pienkowski T, Im YH, et al. Efficacy and safety of neoadjuvant pertuzumab and trastuzumab in women with locally advanced, inflammatory, or early HER2-positive breast cancer (NeoSphere): a randomised multicentre, open-label, phase 2 trial. Lancet Oncol 2012;13:25-32.

5. Early Breast Cancer Trialists' Collaborative Group (EBCTCG). Long-term outcomes for neoadjuvant versus adjuvant chemotherapy in early breast cancer: metaanalysis of individual patient data from ten randomised trials. Lancet Oncol 2018;19:27-39.

6. Mamounas EP. Impact of neoadjuvant chemotherapy on locoregional surgical treatment of breast cancer. Ann Surg Oncol 2015;22:1425-33.

7. Marinovich ML, Macaskill P, Irwig L, et al. Metaanalysis of agreement between MRI and pathologic breast tumour size after neoadjuvant chemotherapy. Br J Cancer 2013;109:1528-36.

8. Scheel JR, Kim E, Partridge SC, et al. MRI, Clinical Examination, and Mammography for Preoperative Assessment of Residual Disease and Pathologic Complete Response After Neoadjuvant Chemotherapy for Breast Cancer: ACRIN 6657 Trial. AJR Am J Roentgenol 2018;210:1376-85.

9. Nakamura S, Ishiyama M, Tsunoda-Shimizu $H$. Magnetic resonance mammography has limited ability to estimate pathological complete remission after primary chemotherapy or radiofrequency ablation therapy. Breast Cancer 2007;14:123-30.

10. Orlando L, Viale G, Bria E, et al. Discordance in pathology report after central pathology review: Implications for breast cancer adjuvant treatment. Breast
2016;30:151-5.

11. Romanoff AM, Cohen A, Schmidt H, et al. Breast pathology review: does it make a difference? Ann Surg Oncol 2014;21:3504-8.

12. Özkurt E, Sakai T, Wong SM, et al. Survival Outcomes for Patients With Clinical Complete Response After Neoadjuvant Chemotherapy: Is Omitting Surgery an Option? Ann Surg Oncol 2019;26:3260-8.

13. Mauri D, Pavlidis N, Ioannidis JP. Neoadjuvant versus adjuvant systemic treatment in breast cancer: a metaanalysis. J Natl Cancer Inst 2005;97:188-94.

14. Alonso-Bartolome P, Ortega Garcia E, Garijo Ayensa F, et al. Utility of the tumor bed marker in patients with breast cancer receiving induction chemotherapy. Acta Radiol 2002;43:29-33.

15. Rea D, Tomlins A, Francis A. Time to stop operating on breast cancer patients with pathological complete response? Eur J Surg Oncol 2013;39:924-30.

16. Shigematsu H, Fujisawa T, Shien T, et al. Omitting surgery for early breast cancer showing clinical complete response to primary systemic therapy. Jpn J Clin Oncol 2020;50:629-34.

17. van la Parra RF, Kuerer HM. Selective elimination of breast cancer surgery in exceptional responders: historical perspective and current trials. Breast Cancer Res 2016;18:28.

18. Gradishar WJ, Anderson BO, Blair SL, et al. Breast cancer version 3.2014. J Natl Compr Canc Netw 2014;12:542-90.

19. Mainiero MB, Cinelli CM, Koelliker SL, et al. Axillary ultrasound and fine-needle aspiration in the preoperative evaluation of the breast cancer patient: an algorithm based on tumor size and lymph node appearance. AJR Am J Roentgenol 2010;195:1261-7.

20. Shien T, Akashi-Tanaka S, Yoshida M, et al. Evaluation of axillary status in patients with breast cancer using thinsection CT. Int J Clin Oncol 2008;13:314-9.

21. Uematsu T, Sano M, Homma K. In vitro high-resolution helical CT of small axillary lymph nodes in patients with breast cancer: correlation of CT and histology. AJR Am J Roentgenol 2001;176:1069-74.

22. Liang X, Yu J, Wen B, et al. MRI and FDG-PET/CT based assessment of axillary lymph node metastasis in early breast cancer: a meta-analysis. Clin Radiol 2017;72:295-301.

23. Boughey JC, Suman VJ, Mittendorf EA, et al. Sentinel lymph node surgery after neoadjuvant chemotherapy in patients with node-positive breast cancer: the ACOSOG Z1071 (Alliance) clinical trial. JAMA 2013;310:1455-61. 
24. Tan VK, Goh BK, Fook-Chong S, et al. The feasibility and accuracy of sentinel lymph node biopsy in clinically node-negative patients after neoadjuvant chemotherapy for breast cancer--a systematic review and meta-analysis. J Surg Oncol 2011;104:97-103.

25. Inokuchi M, Kutomi G, Kijima Y, et al. The Japanese Breast Cancer Society clinical practice guidelines for surgical treatment of breast cancer, 2018 edition. Breast Cancer 2020;27:4-8.

26. lvarado R, Yi M, Le-Petross H, et al. The role for sentinel lymph node dissection after neoadjuvant chemotherapy in patients who present with node-positive breast cancer. Ann Surg Oncol 2012;19:3177-84.

27. Shen J, Gilcrease MZ, Babiera GV, et al. Feasibility and accuracy of sentinel lymph node biopsy after preoperative chemotherapy in breast cancer patients with documented

Cite this article as: Sakai T, Ueno T. Local management after neoadjuvant treatment for breast cancer. Chin Clin Oncol 2020;9(3):34. doi: 10.21037/cco-20-164 axillary metastases. Cancer 2007;109:1255-63.

28. Boileau JF, Poirier B, Basik M, et al. Sentinel node biopsy after neoadjuvant chemotherapy in biopsy-proven nodepositive breast cancer: the SN FNAC study. J Clin Oncol 2015;33:258-64.

29. Kuehn T, Bauerfeind I, Fehm T, et al. Sentinel-lymphnode biopsy in patients with breast cancer before and after neoadjuvant chemotherapy (SENTINA): a prospective, multicentre cohort study. Lancet Oncol 2013;14:609-18.

30. NCI Community Oncology Research Program. CTSU Alliance A011202: A Randomized Phase III Trial Evaluating the Role of Axillry Lymph Node Dissection in Breast Cancer Patients (CT1-3 N1) Who Have Positive Sentinel Lymph Node Disease After Neoadjuvant Chemotherapy. Available online: https://www.swog.org/ clinical-trials/ctsua011202 